\title{
Response factors in intermodality localization under conflict conditions
}

\author{
DAVID H. WARREN \\ University of California, Riverside, California 92521
}

\begin{abstract}
The role of response modality in resolving visual-proprioceptive spatial conflict was evaluated. Subjects responded to either the felt or the seen locations of their forefingers viewed through an 11-deg laterally displacing wedge prism. Four response conditions were used: pointing with the contralateral hand either (1) during target presentation or (2) after target offset, (3) pointing with the ipsilateral (target) hand after target offset, and (4) making a visual localization response after target offset. Using the contralateral hand produced a compromise between vision and proprioception that fell about two-thirds of the way toward the optical location. Using the visual response increased visual influence. Using the ipsilateral response decreased visual influence. It was concluded that visual-proprioceptive interaction in spatial localization is not immune to response modality effects. A possible explanation of the response modality effect, based on differential attention, is discussed.
\end{abstract}

Since the important work of Hay, Pick, and Ikeda (1965), the intermodality conflict paradigm has provided a useful way of studying the relations between visual and proprioceptive perception of spatial location. In this paradigm, discrepant visual and proprioceptive information about a target's location is presented, and the subject indicates either the seen or the felt location of the target. Typically, the target is the subject's own forefinger, and the spatial discrepancy is created by having the subject view his forefinger through a wedge prism which displaces the optical image of the finger to one side. The perceived location is usually some compromise between the physical and the optical location of the finger, and may be interpreted to indicate the relative dependence of perceived location on proprioceptive and visual cues. Generally, subjects weight visual information more heavily than proprioceptive, with the target localized about two-thirds of the way from the physical (and presumed proprioceptive) location toward the displaced optical location (Hay et al., 1965; Pick, Warren, \& Hay, 1969; Warren \& Pick, 1970; Welch, Widawski, Matthews, \& Warren, 1979).

It has become common, following the procedure of Pick et al. (1969), to evaluate localization of the conflict targets with reference to localization of uncon-

This research was supported by a University of California intramural research grant and by Grant RR07010-09 awarded by the Biomedical Research Support Grant Program, Division of Research Resources, National Institutes of Health. I acknowledge gratefully the assistance of Tim McCarthy and Brian Henen in conducting the research. I am indebted to Robert B. Welch and Susan J. Lederman for their constructive criticism of the paper. Requests for reprints should be sent to David H. Warren, Department of Psychology, University of California, Riverside, California 92521. flicted visual and proprioceptive targets. Four conditions are used: (1) unconflicted visual target, (2) unconflicted proprioceptive target, (3) conflict, with instructions to indicate the seen location, and (4) conflict, with instructions to indicate the felt location. In Condition 4 , if visual information does not bias proprioceptive localization, then response should be as in Condition 2. To the extent that vision does bias proprioception, response in Condition 4 should move toward Condition 1. Corresponding statements may be made about proprioceptive bias of vision. These intermodality bias effects are labeled $V(P)$ to refer to visual bias of proprioception and $P(V)$ to refer to proprioceptive bias of vision. Formulas to calculate the bias effects are as follows, where the numbers refer to the numbered conditions above:

$$
\mathrm{V}(\mathrm{P})=\frac{2-4}{2-1} \times 100 \quad \mathrm{P}(\mathrm{V})=\frac{1-3}{1-2} \times 100
$$

The multiplier converts the scores to percentages, such that a low percentage score represents little bias by the conflicting modality and a high percentage score represents a large bias.

In much of the work to date, the subject indicates perceived location by pointing with the unseen contralateral hand, either in the presence of the target or directly after its removal. There is an implicit assumption that the contralateral hand serves as an unbiased indicator of perceived location. That is, it is assumed that the use of a response indicator in one modality does not bias the subject to weight more heavily target information from that modality. Some evidence in support of this assumption exists. In an early study of visual-tactual interaction in size judgments, Rock and Victor (1964) found no 
interaction of response condition with dominance. Whether response involved drawing the perceived size of the object, choosing a match tactually, or choosing a match visually, there was virtually complete visual dominance of tactual information. Similarly, Easton and Moran (1978) compared visual, proprioceptive, and visual plus proprioceptive reponses to curvature in an experiment involving visualproprioceptive conflict. There was no variation in capture as a function of response modality: All response conditions showed the same reliance on visual information.

In contrast, a study by Warren and Pick (Note 1) suggested that response modality affects bias magnitude in the spatial localization paradigm. They reported that when a visual response scale was used, visual dominance of proprioception was complete. The visual response scale was present during the target presentation, however, and it seems possible that the complete visual dominance scores resulted not from a true perceptual judgment, but from the subject's irresistible tendency to choose a visual response location coincident with the optical location of the finger. Warren and Cleaves (1971) compared visual with pointing responses where the response followed, by $3 \mathrm{sec}$, the removal of the target information. Thus the automatic identity responding hypothesized for the Warren and Pick study could not occur. They found significantly greater visual bias of proprioception, and less proprioceptive bias of vision, with the visual than with the pointing response, thus lending support to the notion that response modality interacts with measured bias effects. However, in the pointing condition, the subject used the same hand for response that had served as the target, and thus the apparently greater weight given the proprioceptive information may have resulted from a tendency to recreate a proprioceptive posture, rather than from a true spatial judgment in which proprioceptive information was more heavily weighted.

Kelso, Cook, Olson, and Epstein (1975) suggested an attention-based explanation for the Warren and Cleaves response modality effect. If the subject makes pointing responses, which have a strong proprioceptive component, then he will attend more to the proprioceptive target information, and thus weight it more heavily, than if he were to make visual responses.

Kelso et al. (1975) demonstrated an attentional effect on the course of adaptation to prolonged visualproprioceptive discrepancy, and they hypothesized, although they did not directly show, that the altered adaptation was caused by an altered dominance relationship, itself caused by the attentional manipulation. Warren and Schmitt (1978) failed to alter the normal visual dominance of proprioception by instructing subjects to attend differentially, but succeeded in reversing it by embedding the discrepancy trials within a series of nondiscrepancy trials in which the subject pointed to nonviewed proprioceptive targets. Apparently, the ongoing series of proprioceptive localizations caused the subject to atterid more to the proprioceptive information in the discrepancy trials, thus reducing the usual strong visual dominance of proprioception.

With these lines of evidence suggesting that visual dominance of proprioception can be attenuated, perhaps by attentional factors, it is important to examine the interaction of response modality with visual dominance effects: It is important to know whether the magnitude of the visual dominance effect found with the discrepancy paradigm is dependent on response modality. Although the results of Warren and Cleaves (1971) suggest such dependence, their evidence is equivocal because of the use of the ipsilateral hand for response. In addition, a 3-sec response delay was used in both visual and pointing response conditions, and it is not clear whether this methodological difference from previous studies, in which the pointing response has typically been made while the target information is still present, may account for the lesser dependence on visual information in the Warren and Cleaves results.

The present study evaluated visual bias of proprioception, $\mathrm{V}(\mathrm{P})$, and proprioceptive bias of vision, $\mathrm{P}(\mathrm{V})$, in four response conditions. The simultaneous pointing condition was similar to the method used in most prior research: The subject points with the unseen contralateral hand to the perceived target location while the target information is still present. In the contralateral pointing-delay condition, the subject also points with the unseen contralateral hand, but not until $3 \mathrm{sec}$ after removal of the target information. In the ipsilateral pointing-delay condition, the subject points $3 \mathrm{sec}$ after target removal, but with the hand that served as the target. In the visual-delay condition, the subject chooses a position on a visual scale presented $3 \mathrm{sec}$ after target removal. The first two conditions compare responding (in both cases with the contralateral hand) in the presence and in the absence of target information. Comparison of the visual-delay with the contralateral pointing-delay conditions shows whether proprioceptive information is favored when the target hand, rather than the nontarget hand, is used for pointing.

\section{METHOD}

\section{Subjects}

Fifty-five students from various psychology courses were tested and received either credit toward a research participation requirement or payment. Seven of the subjects, spread across various conditions, were rejected on the basis of extreme bias scores (either greater than $150 \%$ or less than $-50 \%$, according to the 
procedure of Pick et al., 1969). Thus there were 12 subjects in each of the four response conditions. Response condition was a between-groups variable, whereas bias effect (described below) was within subjects.

\section{Procedure}

Each subject was tested individually in a session lasting approximately $20 \mathrm{~min}$. The subject viewed visual targets through a 20-diopter wedge prism oriented with its base to the subject's left, which created an approximately 11-deg rightward displacement of optical target locations. Targets were placed at the subject's straight-ahead and at 5 and 10 deg to either side of straightahead, directly above an opaque shelf that hid the pointing responses. Ten visual and 10 proprioceptive control trials were presented first, with 2 visual and 2 proprioceptive trials at each of the five target locations. The visual control target was a metal peg, viewed through the prism. There was no conflict, since the subject received no proprioceptive information about the target location. The proprioceptive control target was the subject's left forefinger. The subject arrived at the target location by finding the pivot end of a metal rod directly under his chinrest with his left hand and moving his left forefinger out along the rod until it reached a metal stop. Since a shutter in front of the eyepiece remained closed during the proprioceptive control trials, the subject received no conflicting visual information. Thus the control trials served to index the subject's ability to localize visual and proprioceptive targets without intermodality conflict. Half of the subjects localized the 10 proprioceptive targets first, then the 10 visual targets. The remaining subjects received the reverse order.

Following the no-discrepancy control trials were 20 conflict trials. The subject moved his left forefinger out along the rod to find the target location. As soon as he reached the target location, the shutter was raised, allowing him to receive a (displaced) view of his forefinger. On 10 of the trials, the subject was asked to respond to the visual location of the target (visual conflict trials), and on the remaining 10 trials, he was asked to respond to the felt location of the target (proprioceptive conflict trials). The order of conflict trials was counterbalanced, with half of the subjects in each condition performing visual trials before the proprioceptive ones.

The four response conditions (described for the conflict trials) were as follows. Simultaneous pointing: The subject pointed, underneath the opaque shelf, to the target location while the target information was still present. The response was made with the contralateral (right) forefinger, against a curved plastic fence, directly underneath the corresponding fence on which the target finger rested. Contralateral pointing-delay: The target was available for $3 \mathrm{sec}$, then the shutter was dropped and the subject returned his target finger to the chinrest. After $3 \mathrm{sec}$, he responded by pointing to the remembered target location underneath the shelf with his right forefinger. Ipsilateral pointing-delay: The target was available for $3 \mathrm{sec}$, then the shutter was dropped and the subject returned his target finger to the chinrest. After $3 \mathrm{sec}$, he moved the same (left) hand under the shelf and pointed with it to the remembered target location. Visual-delay: The target was available for $3 \mathrm{sec}$, then the shutter was dropped and the subject returned his target finger to the chinrest. During the next $3 \mathrm{sec}$, a visual response scale was lowered to the height at which the target had been placed. The shutter was opened, and the subject responded by choosing the alphanumeric character at the location corresponding to the remembered target location.

For the visual and proprioceptive control trials, corresponding response methods were used. That is, the response was made by pointing with the contralateral hand either simultaneously or with a 3-sec delay, by pointing with the ipsilateral hand with a 3-sec delay, or by choosing a visual scale location with a 3-sec delay.

In all cases, the experimenter read and recorded the response to the nearest degree.

\section{RESULTS}

For each trial, the response location was subtracted from the true target location, giving a signed error score, in degrees, which was positive if the subject responded to his left of the physical target location and negative if he responded to his right. For each subject, a signed mean error was calculated for the proprioceptive control trials, the visual control trials, the visual conflict trials, and the proprioceptive conflict trials. These four means were used to calculate, separately for each subject, a percent visual bias of proprioception score, $\mathrm{V}(\mathrm{P})$, and a percent proprioceptive bias of vision score, $P(V)$, according to the formulas outlined earlier. Standard deviations were also calculated for each of the four types of trials, separately for each subject.

\section{Visual Bias of Proprioception}

The V(P) scores were entered into a one-way analysis of variance, with the four response conditions serving as levels of the variable. The effect of response condition was significant $[F(3,44)=14.35, p<.01]$. The group means appear in Table 1 . The following contrasts were tested. (1) Visual-delay was compared with contralateral pointing-delay. The visual condition produced $\mathrm{V}(\mathrm{P})$ that was significantly greater than the pointing condition $[F(1,44)=7.24, p<.02]$. Clearly, the magnitude of $\mathrm{V}(\mathrm{P})$ is not independent of response modality. (2) Contralateral pointing-delay was compared with ipsilateral pointing-delay. There was a significantly greater $\mathrm{V}(\mathrm{P})$ effect for the contralateral condition $[F(1,44)=14.68, p<.01]$. This

Table 1

Group Mean Percent V(P) and P(V) Scores, and Mean V(P) + P(V), with 95\% Confidence Intervals

\begin{tabular}{|c|c|c|c|c|c|c|}
\hline & \multicolumn{6}{|c|}{ Group Mean Score } \\
\hline & \multicolumn{2}{|c|}{$V(P)$} & \multicolumn{2}{|c|}{$\mathrm{P}(\mathrm{V})$} & \multicolumn{2}{|c|}{$V(P)+P(V)$} \\
\hline & Mean & SD & Mean & SD & Mean & SD \\
\hline Simultaneous Pointing & 58.9 & 9.7 & 34.4 & 9.5 & 93.3 & 3.8 \\
\hline Contralateral Pointing-Delay & 65.0 & 18.0 & 24.0 & 13.3 & 89.0 & 17.4 \\
\hline Ipsilateral Pointing-Delay & 25.3 & 14.7 & 27.8 & 10.1 & 53.0 & 13.0 \\
\hline Visual-Delay & 92.9 & 11.8 & -2.7 & 7.3 & 90.3 & 11.6 \\
\hline
\end{tabular}


finding suggests that the relative strength of proprioceptive information found by Warren and Cleaves (1971) in the pointing response condition was due at least in part to their use of the target hand for response: It may be that the subject in this situation attempts to recreate an arm posture, rather than localize a point in space. (3) Contralateral pointingdelay was compared with simultaneous pointing. There was no difference $[F(1,44)=.34, p>.05]$. The introduction of a short delay between target termination and response does not affect the magnitude of $V(P)$. The result lends further strength to the suggestion that the Warren and Cleaves (1971) results were due to use of the ipsilateral hand for response, rather than to the 3-sec response delay used in that study.

\section{Proprioceptive Bias of Vision}

The $P(V)$ scores were entered into a one-way analysis of variance, with the four response conditions serving as levels of the variable. The effect of response condition was again significant $[\mathrm{F}(3,44)=9.18$, $p<.01]$. The same set of contrasts was tested as in the case of $V(P)$. Visual-delay produced a significantly lower mean $P(V)$ than did contralateral pointing-delay $[F(1,44)=12.33, p<.01]$, indicating, as in the case of $V(P)$, that the use of a visual response tends to increase the role of the visual information in resolving the spatial discrepancy. The remaining contrasts did not produce significant effects.

Thus, the hypothesis that response modality does not interact with intermodality bias effects may be confidently rejected on the basis of both the V(P) and the $P(V)$ analyses. Use of a pointing response led to a greater weighting of proprioceptive information [higher $P(V)$ and lower $V(P)$ ] in resolving the conflict than did the use of a visual response. Clearly, then, intermodality bias data may not be taken to represent an absolute weighting of visual and proprioceptive information. Response modality must be taken into account, although exactly how response modality interacts with intermodality bias effects remains unclear.

\section{Is There a Single Perceived Location?}

Prior research on visual-proprioceptive bias effects (Pick et al., 1969; Warren \& Schmitt, 1978) has shown that the subject tends to respond to the same compromise location on conflict trials whether he is asked for the visual or the felt location of his forefinger. Because of the method of calculation of bias effects, the result is that the two percent bias effects sum to roughly $100 \%$.

Table 1 shows the mean $V(P)+P(V)$ for the four groups, with $95 \%$ confidence intervals. The sums for two conditions are not different from $100 \%$, and that for simultaneous pointing is within the same range and is apparently significantly different from $100 \%$ only by virtue of the very low within-group variance. By contrast, the sum for ipsilateral pointingdelay is clearly far less than $100 \%$. The difference is created by the low mean V(P), compared to the other conditions. Although it is tempting to conclude that the subject had, in contrast to the other conditions, two effectively different targets available, it seems more parsimonious to regard the failure of $V(P)$ and $P(V)$ to add to $100 \%$ as a result of the subject's tendency to recreate an arm posture, as discussed previously, rather than to attempt to localize separate remembered locations in space.

Thus, for the most part, the present results corroborate previous research in suggesting that, in the visual-proprioceptive spatial conflict situation, the subject responds to a single target location rather than to two locations differentiated by modality.

\section{DISCUSSION}

The primary conclusion is that visual-proprioceptive bias effects depend on response modality. Use of a visual response enhances the role of visual information in the subject's resolution of a visual-proprioceptive discrepancy, leading to a greater $V(P)$ and a lesser $P(V)$. Similarly, a pointing response enhances the role of the proprioceptive information, leading to a reduced $V(P)$ and an increased $P(V)$. Thus, any particular value of $V(P)$ or $P(V)$ does not represent an absolute weighting of visual and proprioceptive information in intermodality spatial localization as long as the response is in one of the two modalities. An amodal response procedure, if one could be devised, might reveal absolute weighting.

This conclusion conflicts with those reached by Easton and Moran (1978) and Rock and Victor (1964), who found no effect of response modality on discrepancy judgments. It may be that spatial localization tasks differ in this respect from size and shape tasks. An alternative, and more provocative, suggestion is that, in tasks where visual dominance is virtually complete (Easton \& Moran, 1978; Rock \& Victor, 1964), response modality can have little effect.In spatial localization tasks, where visual dominance is less and proprioceptive information plays a significant role, response modality effects can emerge. This issue should be explored.

The question remains about how this response modality interaction arises. Kelso et al. (1975) suggested that the Warren and Cleaves (1971) results were due to enhanced attention to the information from the response modality, and Warren and Schmitt (1978) present firm evidence that attentional factors can influence the relative weight placed on discrepant visual and proprioceptive information. The results of the present study are consonant with the attention formulation.

Welch et al. (1979) proposed a "modality precision" hypothesis, that the more precisely (i.e., less variably) targets can be localized in one modality 
compared to the other, the more the first modality will bias the other and the less the first will be biased by the other. Comparisons of mean standard deviations for the visual and proprioceptive control tasks from the visual-delay and contralateral pointingdelay conditions reveal the following: In the visualdelay condition, the visual control targets were localized far more precisely (SD $=1.14 \mathrm{deg}$ ) than proprioceptive targets $(\mathrm{SD}=3.64 \mathrm{deg}$ ) and vision biased proprioception heavily in the conflict situation. In the contralateral pointing-delay condition, visual targets were localized about as precisely ( $\mathrm{SD}=2.97 \mathrm{deg}$ ) as proprioceptive targets $(\mathrm{SD}=3.02 \mathrm{deg})$ and $\mathrm{V}(\mathrm{P})$ was significantly weaker than in the visual-delay condition. This set of findings is consonant with the modality precision formulation.

The data thus contradict neither hypothesis. The hypotheses, indeed, are not contradictory. A parsimonious formulation is the following. Attention is drawn to the responding modality, and thus to its location information, more than to the other. This differential allocation of attention has two effects. One is to increase precision of localization of targets in the responding modality. The mean standard deviation values given above bear this out: Visual control targets were localized more precisely in the visualdelay condition than in the contralateral pointingdelay condition $[\mathrm{t}(11)=3.87, \mathrm{p}<.01]$; the pattern was reversed for proprioceptive control targets [ $t(11)$ $=1.58, .05<\mathrm{p}<.10]$. The second, and perhaps entirely independent, effect of attentional allocation to the responding modality is to increase the weight of the responding modality in the conflict situation, as the weight of the evidence from this study clearly shows.

\section{REFERENCE NOTE}

1. Warren, D. H., \& Pick, H. L., Jr. Intermodality interactions: Vision, audition, and proprioception. Paper presented at the meetings of the Midwestern Psychological Association, Chicago, May 1968.

\section{REFERENCES}

Easton, R. D., \& Moran, P. W. A quantitative confirmation of visual capture of curvature. Journal of General Psychology, $1978,98,105-112$.

Hay, J. C., Pick, H. L., JR., \& Ikeda, K. Visual capture produced by prism spectacles. Psychonomic Science, 1965, 2, 215 216.

Kelso, J. A. S., Cook, E., Olson, M. E., \& Epstein, W. Allocation of attention and the locus of adaptation to displaced vision. Journal of Experimental Psychology: Human Perception and Performance, 1975, 1, 237-245.

Pick, H. L., Jr., Warren, D. H., \& Hay, J. C. Sensory conflict in judgments of spatial direction. Perception \& Psychophysics, 1969, 6, 203-205.

Rock, I., \& Victor, J. Vision and touch: An experimentally created conflict between the two senses. Science, 1964, 143, 594596.

Warren, D. H., \& Cleaves, W. T. Visual-proprioceptive interaction under large amounts of conflict. Journal of Experimental Psychology, 1971, 90, 206-214.

Warren, D. H., \& PICK, H. L., JR. Intermodality interactions in localization in blind and sighted people. Perception \& Psychophysics, 1970, 8, 430-432.

WarRen, D. H., \& SChMitT, T. L. On the plasticity of visualproprioceptive bias effects. Journal of Experimental Psychology: Human Perception and Performance, 1978, 4, 302-310.

Welch, R. B., Widawski, M. H., Matthews, J. H., \& WARREN, D. H. An examination of the relationship between visual capture and prism adaptation. Perception \& Psychophysics, 1979, 25, 126-132.

(Received for publication August 15, 1979; revision accepted November $2,1979$. 